\title{
Design and Implementation of Health Monitoring Device using FPGA
}

\author{
Vaibhavi Bhelkar \\ Student, Department of \\ Electronics Engineering \\ A.I.S.S.M.S's \\ Institute of \\ Information Technology \\ Pune, India
}

\author{
D. K. Shedge, PhD \\ Associate Professor, \\ Department of \\ Electronics Engineering \\ A.I.S.S.M.S's \\ Institute of \\ Information Technology \\ Pune, India
}

\begin{abstract}
In recent time's healthcare is gaining importance and technology is playing an important role in patient's health monitoring. Health monitoring deals with monitoring various parameters of patient's continuously over a period of time. Sensors are used for monitoring parameters which in turn sends the output to a central unit for further data processing. A patient's health monitoring device based on FPGA has been developed for monitoring parameters such as heartbeat, temperature and motion. FPGA has been used here instead of microcontroller as it is reconfigurable and necessary hardware can be added as and when required. The graphical user interface (GUI) is developed using visual basic and the data received from these sensors is forwarded to GUI. When the parameters are above the threshold value, email, message will be sent directly to the doctor for remote monitoring. As a first aid to the patient the doctor can reply by prescribing the necessary medicine which the patient can consume after making use of switch button available on the graphical user interface. Android application shows the patients data in real time to the doctor at remote location.
\end{abstract}

\section{Keywords}

Temperature sensor, accelerometer, heartbeat sensor, database

\section{INTRODUCTION}

In recent years heath monitoring is gaining importance. Changes when observed at regular interval of time can be stated as health monitoring. Identification of fluctuations occurring in health parameters can also be stated as health monitoring. Vital parameters should be monitored in such a way that the standard of living of an individual remains unaffected. The monitoring systems should have the provision of providing feedback in real time [5].Taking into consideration the changing demands of monitoring systems various health monitoring systems have been developed in recent times.

With the tremendous growth in he field of information technology, computers and networks they are been used on a large scale in the area of health monitoring. Combination of telecommunication and information technology is used for giving services related to health along with facility of providing services over a distance is done in the field of telemedicine. Mobile phone is a simple example of many other systems which are developed by making use of computers and networks. Condition of patient being monitored at distance can be termed as tele monitoring which deals with monitoring the health parameters of any individual when the doctor and the patient are not at the same location it is more convenient if the patient cannot travel larger distance and when the patient can perform basic heath monitoring tasks by themselves.

Biosensors are the sensors which are used in biomedical applications. Heart rate, body temperature, respiration, blood pressure etc. which are the vital parameters that can be measured by biosensors [1]. Health care technologies re mostly confined to hospitals. Research has been carried out handle the issue of hindrance in the movement of patients. Smartphone can be used as a solution for receiving and transmitting the data. Data transmitted from any system should be saved so that it can be used as needed. Health monitoring systems also require to save their data. Database system can be helpful for saving the data obtained from the health monitoring systems. Here in this paper we have made use of a web based database system where the web server will save the data at regular interval we have made used of MySQL since it's an open source database management system. FPGA is used as a controller through which data is send to graphical user interface(GUI) and then passed on to the server which further sends the data to smartphone which is displayed with the help of android application.

\section{LITERATURE SURVEY}

A mobile device for monitoring pulse and respiration named as "FlexPock" is proposed by making use sensor based on the principal of magnetic induction (MI) and reflective photo plethysmography (rPPG) for measurement of respiration and pulse respectively. Microcontroller is used as the central processing unit and the information is transferred to the mobile phone using the Bluetooth technology. The device works in a non- contact way and can be kept in pocket due its small size and flexibility [5]. Android based patient monitoring is proposed in [7] this system can detect multi parameters of the body such as ECG, heart rate, blood pressure, temperature and pulse oximeter this system has advantages such as it is portable, mobile, compact, consumes less power, stores the data in database. Monitoring of parameters such as ECG, heart rate, $\mathrm{SpO}$ 2, pulse rate and temperature are measured using a patient monitoring system described in prema.in the system described here the values are entered into a database and are uploaded into a web based server manually. A system was developed in [3] which describes a mobile health monitoring system consisting of a portable multifunctional physiological parameters detecting 3AHcare node and a real-time data telemetry using mobile program on the smartphone with the help android operating system. The data of this system is stored locally on micro SD flash and data transmission is done with the help of TCP/IP protocol. A multi-parameter patient monitoring system for method for ICU patients based on GSM has been presented in 
[2]. It also has facility of controlling the dosage of medicine of the patient. Communication within the hospital and through SMS has been done by using GSM. A system monitoring blood pressure, pulse rate and fall of an elderly at home has been described in [8]. For analysis of data in real time a smart phone/laptop based network was used. Bluetooth technology is used for transfer of data from sensors. A system making use of heart rate monitor for processing the data to smartphone with the ability of calculating energy consumption, detecting states of stress and classifying events in arrhythmia, the devices have advantages such as being portable, economical, easy to use etc. and useful for people with heart diseases and neurological disorders. The device proposed by them does not make use of traditionally used multichannel electrocardiograph instead they have used hear rate monitor [6].

\section{PROPOSED SYSTEM}

Most of the systems makes use of microcontroller here we are making use of FPGA as it has some advantages over the conventionally used microcontroller such as FPGA is reconfigurable, flexible functionality can be added as and when needed, adoption for advanced if required, better power efficiency, small operations when required at precise time, real time requirements, improved performance. The block diagram of the proposed system is as follow:

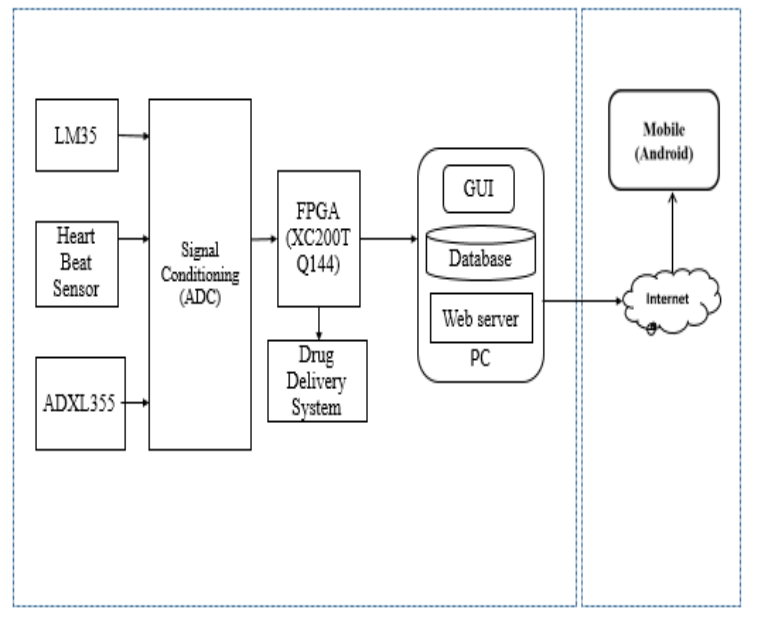

Figure 1: Block diagram of proposed health monitoring device

The requirements for implementation of the proposed system are as listed below:

\subsection{Hardware used}

- $\quad$ SPARTAN 3 (XC3200TQ144)

- $\quad$ LM35 sensor

- Heartbeat sensor

- Accelerometer ADXL355

- Personal computer

- Android mobile phone

\subsection{Software used \\ - Visual basic \\ - $\quad$ Xilinx}

- Geanymotion

- $\quad$ Android studio

- Apache

- MySQL database

- $\quad$ PHP

\subsection{Patient parameters}

\subsubsection{Temperature}

Body temperature is one of the vital signs for assessment of patient's health. Fluctuations occur in body temperature in case of infection, inflammation, heart attack, shocks and so on. Thermometer is used for traditionally for measuring body measurements but now getting replaced temperature sensors and its similar electrical and electronic methods [4].The commonly accepted average core body temperature is $37.0{ }^{\circ} \mathrm{C}$ (98.6 OF).

\subsubsection{Heart rate}

Another important physiological parameter is heart rate. There are fluctuations in the heat rate due to illness, injury, exercise etc. Monitoring heart rate can help in identification of health related issues. Heart rate is also known as the pulse rate. Heart rate sensor is used to give digital output of heartbeat when finger is placed on it.

\subsubsection{Acceleration}

The accelerometer can be used to measure gravity in tilt sensing applications as well dynamic acceleration which is result of motion, shock or vibration. The accelerometer used here can give complete 3 -axis acceleration with signal conditioned voltage output.

\section{METHODOLOGY}

The sensors used for measurement of patients parameters are temperature sensor (LM35) the advantage of using LM35 is that it is more accurate in thermistor and it gives higher output than thermocouple does reducing the need of output to be amplified. The heartbeat sensor used her works on the principle of light modulation by blood flow through finger at each pulse. The motion sensor used is ADXL335 which is an energy efficient, compact, and inexpensive device and can be measures the 3 -axis acceleration with a range of $\pm 3 \mathrm{~g}$. All the sensors used are analog sensor so they give analog output and for FPGA (XC200TQ144) SPARTAN3 or any digital device these signal should be converted into digital form. FPGA lacks inbuilt ADC (Analog to digital converter) so extra circuitry is added named as signal conditioning which convert analog signal into digital form for FPGA. The ADC used here is ADC0808 which as an 8bit ADC. The signals or values are processed and checked whether the values are normal or changing in accordance with the threshold values. The values are sent to PC via serial communication, GUI is made using Visual Basic which displays the medical parameters. When the patient requires medicine there are switch buttons on GUI which when pressed can drop medicine at patient's side. An email and SMS will be sent to the doctor when the parameters are in danger. The data from the sensors can be saved on the web server using the save button available on the GUI. The database will be created on the web server by using the MySQL database using the APACHE software. The android application is developed by using Android studio and the application is tested by using Geanymotion emulator. The data from the webserver is seen on the android application in real time. Here PHP has been used as a server side scripting language and also for transferring the data from webserver to 
mobile phone so that the doctor can access the data on his application.

\section{RESULTS}

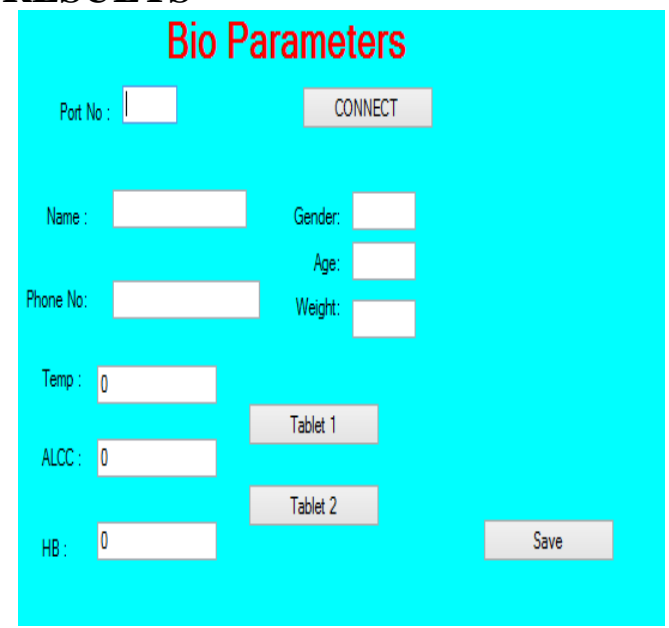

Figure 2: GUI Display

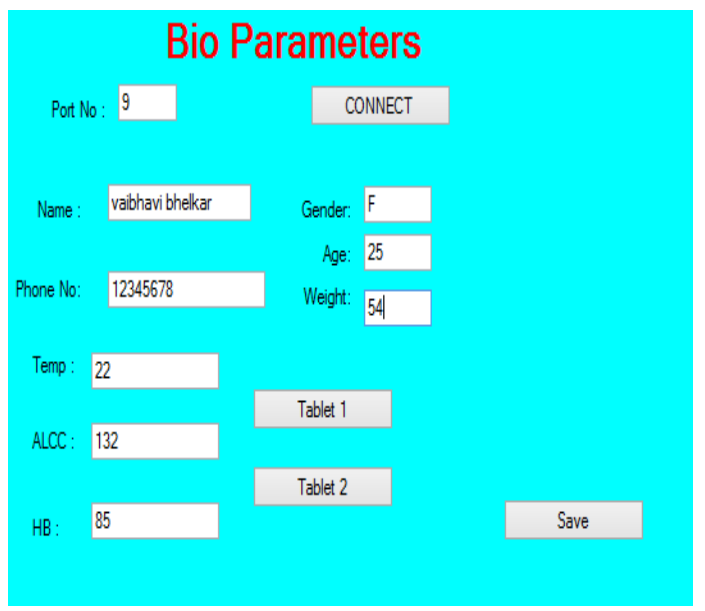

Figure 3: New registration with patient details

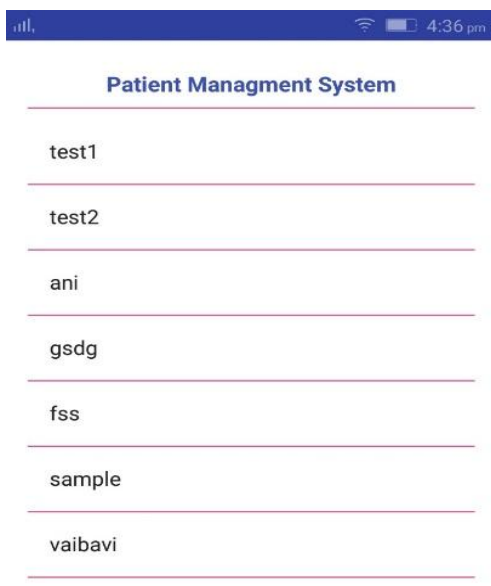

John Regel

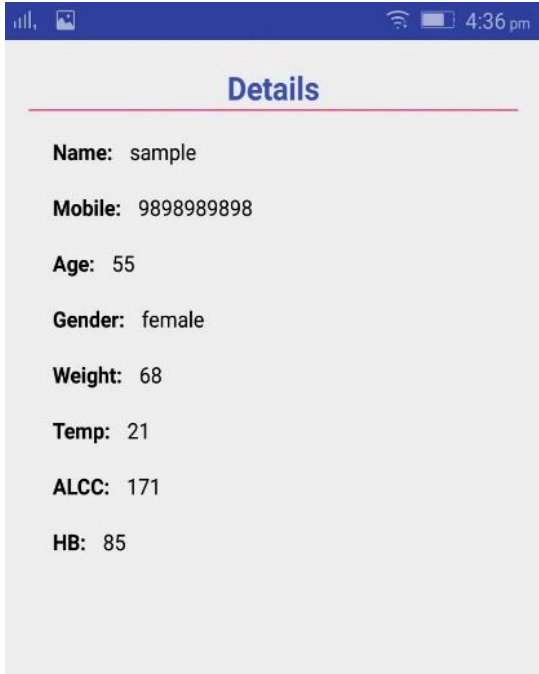

Figure 4: Android application display
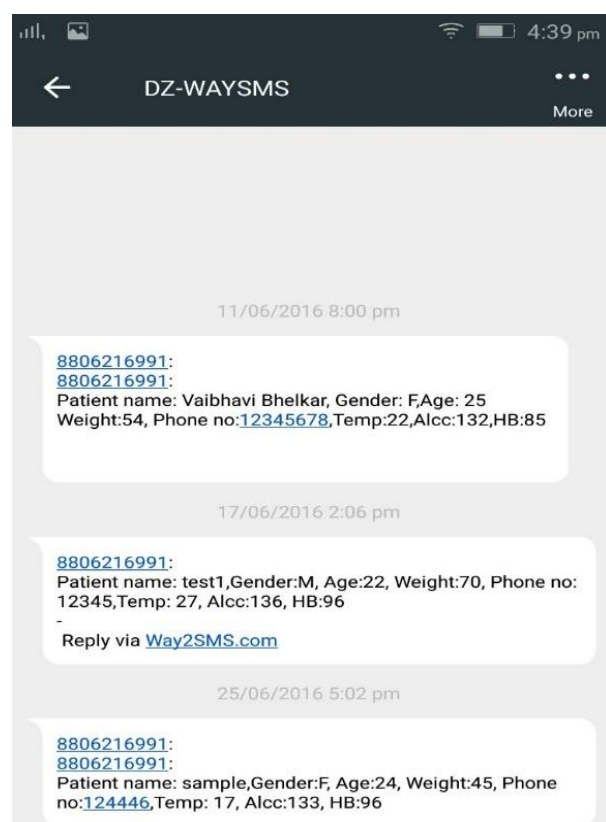

Figure 5: Emergency SMS alert

kannannaikar13@gmail.com to me

20 May View details

Temp value is high

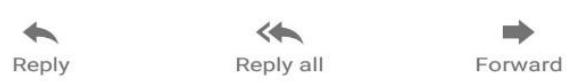

Figure 6: Email service 


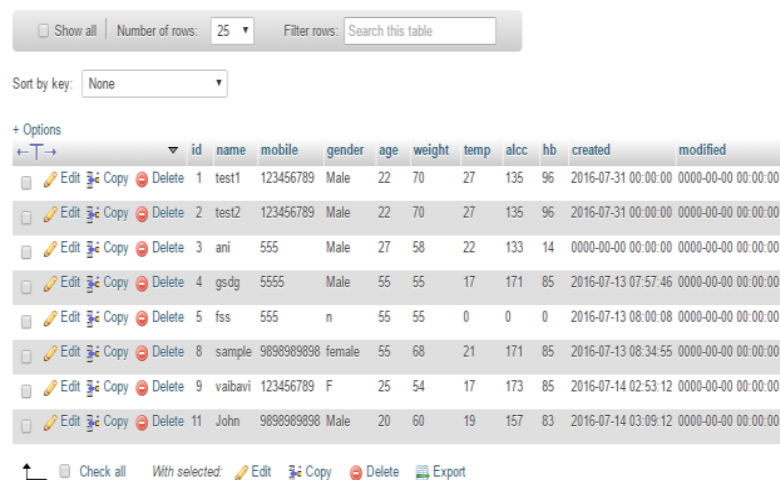

Figure 7: Database

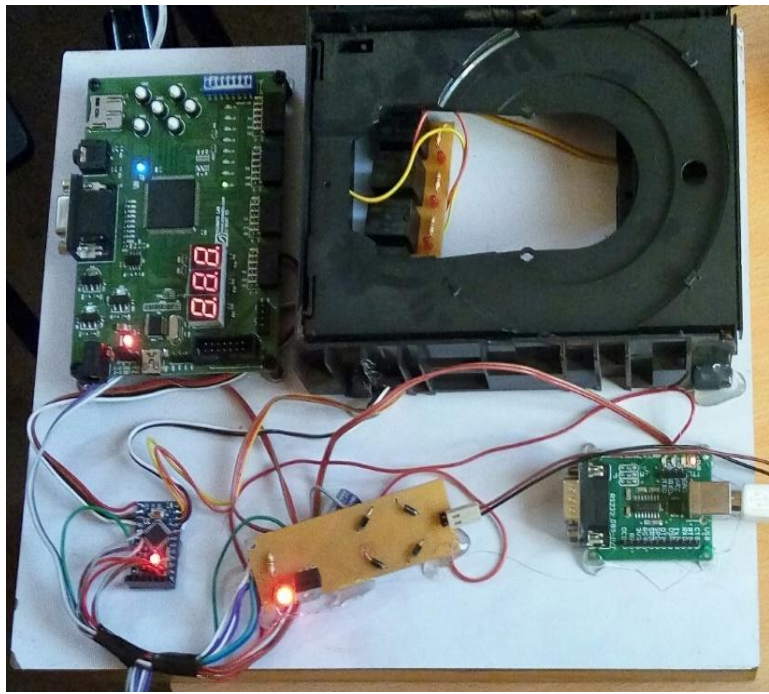

Fig 8 Hardware of the system

\section{CONCLUSIONS AND FUTURE SCOPE}

The patient can see the parameter values with the help of GUI and the drug delivery system is useful when the medicines are already prescribed. SMS and Email systems are useful in case of emergency. Android phones are easily available for doctors and android application is easy to use for accessing the data. Database information is stored for long term use so that data can be accessed at any time from remote area. The system is useful for patients, doctors and medical recording. In future more sensors can be added to the system and different communication technologies can be used for transmitting and receiving the data. An application specific IC can be developed moreover the system can be extended to monitor the motion patterns and visual detection of elderly patients. Accelerometer sensor can be attached to human arm for determining patient's movements and 3D cameras can be used for visual monitoring of elderly patients according to the walking patterns.

\section{ACKNOWLEDGEMENTS}

The motivation factor for this work was the inspiration given by honorable principal Dr. P.B.Mane. The author would also like to thank PG Coordinator Mr.S.B.Dhonde for his immense help and numerous suggestions.

\section{REFERENCES}

[1] Subhas Chandra Mukhopadhyay, "Wearable Sensors for Human Activity Monitoring: A Review", IEEE SENSORS JOURNAL, VOL. 15, NO. 3, pp 1321-1330, MARCH 2015.

[2] Nitin P. Jain, Preeti N. Jain, Trupti P. Agarkar, "An Embedded, GSM based, Multiparameter, Realtime Patient Monitoring System and Control An Implementation for ICU Patients", IEEE, pp. 987992,2012

[3] DongdongLoua, Xianxiang Chena, Zhan Zhaoa, Yundong Xuana, ZhihongXua, HuanJina, XingzuGuo, Zhen Fanga,"A Wireless Health Monitoring System based on Android Operating System", Science Direct, pp. 208215,2013

[4] Michael R. Neuman, "Measurement of Vital Signs:Temperature",IEEE PULSE SEPTEMBER /OCTOBER 2010

[5] Daneil Teichmann, Dennis De Matteis, T horsten Bartelt, Marian Walter and Steffen Leonhardt,“ A Bendable and Wearable Cardiorespiratory Monitoring Device Fusing Two Noncontact Sensor Principles", IEEE JOURNAL OF BIOMEDICAL AND HEALTH INFORMATICS, VOL 19,NO.3, p no 784-793,MAY2015.

[6]Paola Pierleoni, Luca Pernini, Alberto Belli and Lorenzo Palma, "An Android-Based Heart Monitoring System for the Elderly and for Patients with Heart Disease",HINDAWI, International Journal of Telemedicine and Applications Volume, pp1-12, 2014.

[7] Deep Modi, JigneshVyas, Priyank Shah, "Android Based Patient Monitoring System", International Journal For Technological Research In Engineering Volume 1, Issue 9, ISSN (Online): 2347 - 4718 , May-2014

[8] Nitha V Panicker, Sukesh Kumar A, "Development of a Blood Pressure Monitoring System for Home health Application", International Conference on Circuit, Power and Computing Technologies [ICCPCT], 2015 